\title{
ON A NEW GEOMETRICAL DERIVATION OF TWO-DIMENSIONAL FINSLER MANIFOLDS WITH CONSTANT MAIN SCALAR
}

\author{
Sz. VATtamány and Cs. Vincze
}

\begin{abstract}
In this paper we investigate the problem what kind of (two-dimensional) Finsler manifolds have a conformal change leaving the mixed curvature of the Berwald connection invariant? We establish a differential equation for such Finslerian energy functions and present the solutions under some simplification. As we shall see they are essentially the same as the singular Finsler metrics with constant main scalar determined by L. BERWALD.
\end{abstract}

\section{Introduction}

In this short note we are going to determine all Finsler manifolds of dimension 2 which admit a conformal change such that the mixed curvature tensor of the Berwald connection is invariant. It is well-known that the vanishing of the mixed curvature characterizes the so-called Berwald manifolds; see e.g. [7] and [10]. Geometrically this means that the canonical horizontal distribution (nonlinear connection) arises from a linear connection on the underlying manifold.

The conformal equivalence of Finsler manifolds is just the same as it is in Riemannian case, that is the metrical structures differ in a proportional function. The basic transformation formulas are summarized in Hashiguchi's classical paper [3]. The mixed curvature tensor (as the most of the others) is far away from being conformally invariant. Consequently, it is an interesting question whether there are conformally equivalent Berwald manifolds. This problem was originally posed in Matsumoto's paper [8]. Here we deal with a more general case prescribing only the invariance of the mixed curvature which is not necessarily zero. Our main result is the reproduction of Berwald's list of two-dimensional Finsler manifolds with constant main scalar as particular solutions.

In case of dimension $n \geq 3$ the problem is solved in [13]: the invariance of the mixed curvature implies that the Finslerian energy function must be of the form $\mathbf{A}$ by the classification given in Section 3. We have to emphasize that these geometrical structures are always singular. More precisely, the condition of differentiability is not satisfied along a distinguished ray in each tangent space.

1991 Mathematics Subject Classification. 53C60, 58B20.

Key words and phrases. Finsler manifold, conformal change, mixed curvature.

Typeset by $\mathcal{A}_{\mathcal{M}} \mathcal{S}-\mathrm{T}_{\mathrm{E}} \mathrm{X}$ 


\section{Preliminaries}

2.1. Finsler manifolds. Let $M$ be a differentiable manifold equipped with a function $L: T M \rightarrow \mathbb{R}$ such that

(i) $\forall v \in T M \backslash\{0\}: L(v)>0$ and $L(0)=0$.

(ii) $L$ is homogeneous of degree 1, i.e. $\forall t \in \mathbb{R}^{+}: L(t v)=t L(v)$.

(iii) $L$ is continuous on the tangent manifold $T M$ and smooth except the zero section.

(iv) The fundamental form $\omega:=d d_{J} E$ is nondegenerate, where $E:=\frac{1}{2} L^{2}$ is the so-called energy function.

The Riemann-Finsler metric of the Finsler manifold $(M, L)$ is defined by the formula

$$
g(J X, J Y):=\omega(J X, Y),
$$

where $X, Y$ are vector fields on $T M$ and $J$ is the canonical almost tangent structure on the tangent bundle $\pi: T M \rightarrow M$; for the details see [6]. The tensor field

$$
\mathcal{C}_{b}(X, Y, Z):=\frac{1}{2}(J X g(J Y, J Z)-g(J[J X, Y], J Z)-g(J Y, J[J X, Z]))
$$

is the lowered Cartan tensor of the Finsler manifold.

2.2. Two Finsler manifolds $(M, L)$ and $(M, \tilde{L})$ are said to be conformally equivalent if there exists a positive smooth function $\varphi: T M \backslash\{0\} \rightarrow \mathbb{R}$ such that

$$
\tilde{g}=\varphi g .
$$

In this case we also speak of the conformal change of the metric $g$. It is well-known due to M.S. Knebelman that $\varphi$ can be always written in the form

$$
\varphi=\exp \circ \alpha^{v},
$$

where $\alpha^{v}:=\alpha \circ \pi$ is the vertical lift of the function $\alpha: M \rightarrow \mathbb{R}$. For this reason the changed metric will be denoted by $g_{\alpha}$ instead of $\tilde{g}$.

Remark. Using the special form of the scale function, it can be easily seen that the Cartan tensor $\mathcal{C}$ defined by the formula

$$
g(\mathcal{C}(X, Y), J Z)=\mathcal{C}_{b}(X, Y, Z)
$$

is invariant under any conformal change; see [3] and [9].

2.3. Since the fundamental form is nondegenerate there exists a unique vector field $S$ on the tangent manifold satisfying the condition

$$
\iota_{S} \omega=-d E
$$

this vector field is called the canonical spray. It generates a horizontal distribution (nonlinear connection) $h$ in a usual manner

$$
h:=\frac{1}{2}(1+[J, S])
$$


see [4], [5] and [6]. The Berwald connection is one of the classical Finsler connections induced by the canonical horizontal distribution. Using the standard technical tools of tangent bundle geometry such as vertical, complete and horizontal lifts $X^{v}, X^{c}$ and $X^{h}$ of a vector field $X \in \mathfrak{X}(M)$ the mixed curvature tensor (called as $h v$ curvature in [7]) of the Berwald connection can be calculated as follows

$$
\stackrel{\circ}{\mathbb{P}}\left(X^{c}, Y^{c}\right) Z^{c}=\left[\left[X^{h}, Y^{v}\right], Z^{v}\right] .
$$

The vanishing of $\stackrel{\circ}{\mathbb{P}}$ implies that the vector field $\left[X^{h}, Y^{v}\right]$ is a vertical lift, i.e. it can be interpreted as the covariant derivative of the vector field $Y$ with respect to a linear connection on the underlying manifold.

\section{The discussion of the problem}

Suppose that the mixed curvature tensor $\stackrel{\circ}{\mathbb{P}}_{\alpha}$ of the changed metric coincides the mixed curvature tensor $\stackrel{\circ}{\mathbb{P}}$ of the metric $g$ and let us denote by $h_{\alpha}$ and $h$ the corresponding canonical nonlinear connections. We have

$$
\begin{aligned}
0 & =\stackrel{\circ}{\mathbb{P}} \alpha\left(X^{c}, Y^{c}\right) Z^{c}-\stackrel{\circ}{\mathbb{P}}\left(X^{c}, Y^{c}\right) Z^{c}=\left[\left[X^{h_{\alpha}}, Y^{v}\right], Z^{v}\right]-\left[\left[X^{h}, Y^{v}\right], Z^{v}\right] \\
& =\left[\left[X^{h_{\alpha}}, Y^{v}\right]-\left[X^{h}, Y^{v}\right], Z^{v}\right],
\end{aligned}
$$

which means that the vector field $\left[X^{h_{\alpha}}, Y^{v}\right]-\left[X^{h}, Y^{v}\right]$ is a vertical lift; see e.g. [10] and [12]. Therefore, as an easy local calculation shows, the components of the difference tensor $h_{\alpha}-h$ are linear and, consequently, the difference of the associated sprays $S_{\alpha}$ and $S$ is a quadratic vector field. As it is well-known (see e.g. [9])

$$
S_{\alpha}=S-\alpha^{c} C+E g r a d \alpha^{v},
$$

where $\alpha^{c}:=S \alpha^{v}$ is the complete lift of $\alpha$ and the gradient vector field is defined by the formula $i_{\text {grad }} v \omega=d \alpha^{v}$. Evaluating both sides on the function $\alpha^{c}$ we have

$$
E\left\|\operatorname{grad} \alpha^{v}\right\|^{2}=\left(S_{\alpha}-S\right) \alpha^{c}+\left(\alpha^{c}\right)^{2},
$$

where the function on the right-hand side is quadratic. This means that it can be considered as a (local) Riemannian energy function $E_{R}$ under the regularity condition $d_{p} \alpha \neq 0, p \in M$.

Let $\operatorname{grad}_{R} \alpha$ and $\operatorname{grad}_{R}^{v} \alpha$ be the Riemannian gradients of the function $\alpha$ and its vertical lift, respectively. For the sake of simplicity we set

$$
J X:=\operatorname{grad} \alpha^{v}, \quad Y^{v}:=\operatorname{grad}_{R}^{v} \alpha .
$$

Since the manifold is of dimension 2, the vector fields $J X, Y^{v}$ and the Liouville vector field $C$ are linearly dependent.

Consider the integral curve $c(t):=v+t Y(p)$ of the vector field $Y^{v}$ under the initial condition $\alpha^{c}(v)=0$ which means that $v$ is tangential to the level hypersurface with respect to $\alpha$ passing through the point $p$. Evaluating the Gram-determinant of $J X, Y^{v}$ and $C$ with respect to $g$, we have that

$$
\begin{gathered}
\left(2 E\|J X\|^{2}\left\|Y^{v}\right\|^{2}+2 g\left(J X, Y^{v}\right) g\left(Y^{v}, C\right) g(J X, C)-g^{2}(J X, C)\left\|Y^{v}\right\|^{2}\right. \\
\left.-g^{2}\left(Y^{v}, C\right)\|J X\|^{2}-2 E g^{2}\left(J X, Y^{v}\right)\right) \circ c=0 . \\
3
\end{gathered}
$$


Here, as an easy calculation shows,

$$
\begin{aligned}
& E\|J X\|^{2}=E_{R}, \quad\left\|Y^{v}\right\|^{2}=Y^{v}\left(Y^{v} E\right), \quad g\left(J X, Y^{v}\right)=(Y \alpha) \circ \pi, \\
& g\left(Y^{v}, C\right)=Y^{v} E, \quad g(J X, C)=\alpha^{c}
\end{aligned}
$$

and we arrive at the following differential equation:

$2\left(a^{2}+t^{2} b^{2}\left(1-b^{2}\right)\right) y(t) y^{\prime \prime}(t)-\left(a^{2}+t^{2} b^{2}\right)\left(y^{\prime}(t)\right)^{2}+4 t b^{4} y(t) y^{\prime}(t)-4 b^{4}(y(t))^{2}=0$, where $y:=E \circ c, a^{2}=2 E_{R}(v)$ and $b^{2}=2 E_{R}(Y(p))$. Using the Cauchy-Schwarz inequality it follows that $0<b^{2} \leq 1$. Introducing $z=\frac{y^{\prime}}{y}$ we obtain a Riccati-type equation:

$$
\left(a^{2}+t^{2} b^{2}\left(1-b^{2}\right)\right) z^{\prime}(t)+\frac{1}{2}\left(a^{2}+t^{2} b^{2}\left(1-2 b^{2}\right)\right)(z(t))^{2}+2 t b^{4} z(t)-2 b^{4}=0 .
$$

If the constants $a$ and $b$ are arbitrary, to find the solutions seems to be very complicated. So we discuss only the extremal case $b^{2}=1$ under the initial condition $z(0)=\frac{K}{a}$, where $K$ is a real parameter with the property $K_{t v}=K_{v}$ for any positive real number $t$. Then the solution has the form

$$
z(t)=2 \frac{2 t+K a}{2 t^{2}+t K a+2 a^{2}}=\frac{2 t+K a}{\left(t+\frac{K a}{4}\right)^{2}+a^{2}\left(1-\frac{K^{2}}{16}\right)} .
$$

(A) If $K^{2}<16$, then

$$
\begin{gathered}
E \circ c(t)=4 K^{*}\left(E_{R} \circ c(t)+K L_{R}(v) \frac{t}{4}\right) \\
\exp \frac{2 K}{\sqrt{16-K^{2}}}\left(\arctan \frac{4 t+K L_{R}(v)}{L_{R}(v) \sqrt{16-K^{2}}}-\arctan \frac{2 K}{\sqrt{16-K^{2}}}\right) .
\end{gathered}
$$

(B) If $K^{2}=16$, then

$$
E \circ c(t)=K^{*}\left(t+\frac{K L_{R}(v)}{4}\right)^{2} \exp \frac{-2 K L_{R}(v)}{4 t+K L_{R}(v)} .
$$

(C) If $K^{2}>16$, then

$$
\begin{gathered}
E \circ c(t)=K^{*}\left(t+\frac{L_{R}(v)}{4}\left(K-\sqrt{K^{2}-16}\right)\right)^{\left(1+\frac{K}{\sqrt{K^{2}-16}}\right)} \\
\left(t+\frac{L_{R}(v)}{4}\left(K+\sqrt{K^{2}-16}\right)\right)^{\left(1-\frac{K}{\sqrt{K^{2}-16}}\right)} .
\end{gathered}
$$


Remark. Consider a local orientation around the point $p \in M$ and let us define the parameter $K$ as follows

$$
K_{v}:= \begin{cases}k_{p}, & \text { if } v \text { and } Y(p) \text { form a positively oriented basis; } \\ -k_{p}, & \text { otherwise. }\end{cases}
$$

Under these conditions the classification $\mathbf{A}, \mathbf{B}$ and $\mathbf{C}$ is just the Berwald's list of two-dimensional Finsler manifolds with a main scalar $k_{p}$ depending only on the position; see [1], formulas 118 I-III; see also [2]. It is well-known that they are Berwald manifolds if and only if the parameter $k$ is a universal constant; for the details see [2] and [11].

According to the previous discussion if a two-dimensional Finsler manifold admits a conformal change such that the mixed curvature remains invariant then the energy function must be of the form $\mathbf{A}, \mathbf{B}, \mathbf{C}$ or further (implicit) solutions of the differential equation. In what follows we shall prove that in the presented explicit cases the mixed curvature is invariant under any conformal change of the metric. Throughout the proof we use the terminology and conventions described in [11]. For the first step consider the so-called Berwald frame $\left(C_{0}, X_{0}, S_{0}, F X_{0}\right)$, where $C_{0}:=\frac{1}{L} C$ is the normalized Liouville vector field, $S_{0}:=\frac{1}{L} S$ and $F$ is the almost complex structure associated with the canonical horizontal distribution. The vector field $X_{0}$ is defined by the help of the usual Gram-Schmidt process such that the quadruple $\left(C_{0}, X_{0}, S_{0}, F X_{0}\right)$ is a (local) orthonormal frame with respect to the prolonged metric $g_{h}$. Since the manifold is of dimension 2 all of geometrical objects can be characterized in terms of the Berwald frame. If

$$
\lambda:=\mathcal{C}_{b}\left(F X_{0}, F X_{0}, F X_{0}\right)
$$

then the function $I:=L \lambda$ is said to be the main scalar of the Finsler manifold in Berwald's sense. Consider now the conformal change $g_{\alpha}=\varphi g$; then

$$
g_{\alpha}\left(\frac{X_{0}}{\sqrt{\varphi}}, \frac{X_{0}}{\sqrt{\varphi}}\right)=\frac{\varphi}{\varphi} g\left(X_{0}, X_{0}\right)=1,
$$

therefore $X_{0 \alpha}=\frac{X_{0}}{\sqrt{\varphi}}$ and, in a similar way $C_{0 \alpha}=\frac{C_{0}}{\sqrt{\varphi}}$. Since

$$
J \circ\left(F_{\alpha}-F\right) \circ J=0 \Rightarrow J\left(F_{\alpha} X_{0 \alpha}-F X_{0 \alpha}\right)=0,
$$

we have that

$$
\begin{aligned}
L_{\alpha} \lambda_{\alpha} & =L_{\alpha} g_{\alpha}\left(\mathcal{C}_{\alpha}\left(F_{\alpha} X_{0 \alpha}, F_{\alpha} X_{0 \alpha}\right), X_{0 \alpha}\right)=L_{\alpha} g_{\alpha}\left(\mathcal{C}\left(F X_{0 \alpha}, F X_{0 \alpha}\right), X_{0 \alpha}\right) \\
& =\varphi^{\frac{3}{2}} g\left(\mathcal{C}\left(\frac{F X_{0}}{\sqrt{\varphi}}, \frac{F X_{0}}{\sqrt{\varphi}}\right), \frac{X_{0}}{\sqrt{\varphi}}\right)=L \lambda,
\end{aligned}
$$

i.e. the main scalar in Berwald's sense is conformally invariant; see also [3], Proposition 1.2 . 
Theorem. If a two-dimensional Finsler manifold has a main scalar depending only on the position then the mixed curvature of the Berwald connection is invariant under any conformal change.

Proof. By the formula (24a) of our previous paper [11]

$$
\stackrel{\circ}{\mathbb{P}}\left(F X_{0}, F X_{0}\right) F X_{0}=-\left[F X_{0}(\lambda)+X_{0}(S \lambda)\right] X_{0}+2 \frac{S \lambda}{L} C_{0}
$$

and, since the main scalar $L \lambda$ has the special form $L \lambda=k \circ \pi$, it follows that

$$
\stackrel{\circ}{\mathbb{P}}\left(F X_{0}, F X_{0}\right) F X_{0}=-\frac{1}{L}\left[F X_{0}\left(k^{v}\right)+X_{0}\left(k^{c}\right)\right] X_{0}+2 \frac{k^{c}}{L^{2}} C_{0} .
$$

On the other hand

$$
\stackrel{\circ}{\mathbb{P}}\left(F_{\alpha} X_{0 \alpha}, F_{\alpha} X_{0 \alpha}\right) F_{\alpha} X_{0 \alpha}=\stackrel{\circ}{\mathbb{P}}\left(F X_{0 \alpha}, F X_{0 \alpha}\right) F X_{0 \alpha}=\frac{1}{\sqrt{\varphi^{3}}} \stackrel{\circ}{\mathbb{P}}\left(F X_{0}, F X_{0}\right) F X_{0} .
$$

Using the invariance of the main scalar, a similar reasoning gives that

$$
\begin{aligned}
& \stackrel{\circ}{\mathbb{P}}_{\alpha}\left(F_{\alpha} X_{0 \alpha}, F_{\alpha} X_{0 \alpha}\right) F_{\alpha} X_{0 \alpha}=-\frac{1}{L_{\alpha}}\left[F_{\alpha} X_{0 \alpha}\left(k^{v}\right)+X_{0 \alpha}\left(k^{c}\right)\right] X_{0 \alpha}+2 \frac{k^{c}}{L_{\alpha}^{2}} C_{0 \alpha} \\
& =-\frac{1}{L_{\alpha}}\left[F X_{0 \alpha}\left(k^{v}\right)+X_{0 \alpha}\left(k^{c}\right)\right] X_{0 \alpha}+2 \frac{k^{c}}{L_{\alpha}^{2}} C_{0 \alpha} \\
& =-\frac{1}{\sqrt{\varphi^{3}} L}\left[F X_{0}\left(k^{v}\right)+X_{0}\left(k^{c}\right)\right] X_{0}+2 \frac{k^{c}}{\sqrt{\varphi^{3}} L^{2}} C_{0}=\frac{1}{\sqrt{\varphi^{3}}} \stackrel{\circ}{\mathbb{P}}\left(F X_{0}, F X_{0}\right) F X_{0} \\
& =\stackrel{\circ}{\mathbb{P}}\left(F_{\alpha} X_{0 \alpha}, F_{\alpha} X_{0 \alpha}\right) F_{\alpha} X_{0 \alpha} .
\end{aligned}
$$

\section{REFERENCES}

[1] L. Berwald, Über zweidimensionale allgemeine metrische Räume, J. reine angew. Math. 156 (1927), 191-210 and 211-222.

[2] L. Berwald, On Finsler and Cartan Geometries III, Two-dimensional Finsler spaces with rectilinear extremals, Ann. of Math. 42 (1941), 84-112.

[3] M. Hashiguchi, On conformal transformations of Finsler metrics, J. Math. Kyoto Univ. 16-1 (1976), 25-50.

[4] J. Grifone, Structure presque tangente et connexions I, Ann. Inst. Fourier Grenoble 22 (1) (1972), 287-334.

[5] J. Grifone, Structure presque tangente et connexions II, Ann. Inst. Fourier Grenoble 22 (3) (1972), 291-338.

[6] M. De Leon and P. R. Rodrigues, Methods of Differential Geometry in Analytical Mechanics, North-Holland, Amsterdam, 1989.

[7] M. Матsumoto, Foundations of Finsler geometry and special Finsler spaces, Kaisheisha Press, Otsu, 1986.

[8] M. Matsumoto, Conformally Berwald and conformally flat Finsler spaces, Publ. Math. Debrecen 58 (1-2) (2001), 275-285.

[9] J. Szilasi and Cs. Vincze, On conformal equivalence of Riemann-Finsler metrics, Publ. Mat. Debrecen 52 (1-2) (1998), 167-185.

[10] J. Szilasi And Cs. Vincze, A new look at Finsler connections and special Finsler manifolds, www.emis.de/journals AMAPN 16 (2000), 33-63.

[11] Sz. Vattamány and Cs. Vincze, Two-dimensional Landsberg manifolds with vanishing Douglas tensor, Ann. Univ. Sci. Budapest. 44 (2001), 11-26. 
[12] Cs. Vincze, On $\mathcal{C}$-conformal changes of Riemann-Finsler metrics, Rend. Circ. Mat. Palermo Serie II 59 (1999), 221-228.

[13] Cs. VincZE, On conformal equivalence of Berwald manifolds all of whose indicatrices have positive curvature, SUT Journal of Math. 39 No.1 (2003), 15-40.

Institute of Mathematics, University of Debrecen, H-4010 Debrecen P.O. Box 12, HUNGARY

E-mail address: csvincze@math.klte.hu, vattam@freemail.hu 